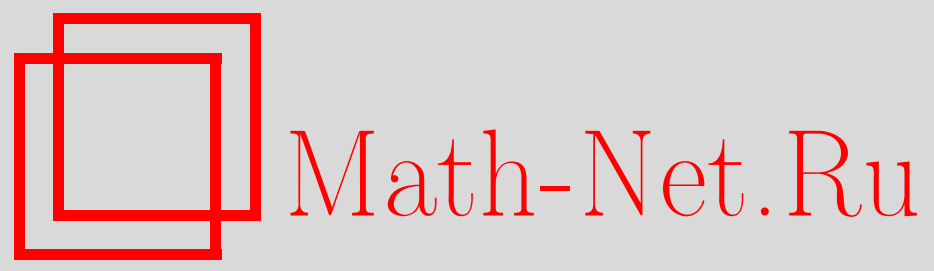

С. А. Алдашев, Корректность локальной краевой задачи в цилиндрической области для многомерного волнового уравнения, Вестн. Сам. гос. техн. ун-та. Сер. Физ.-мат. науки, 2012, выпуск 4(), 48-55

DOI: https://doi.org/10.14498/vsgtu1078

Использование Общероссийского математического портала Math-Net.Ru подразумевает, что вы прочитали и согласны с пользовательским соглашением

http://www . mathnet.ru/rus/agreement

Параметры загрузки:

IP : 54.224 .135 .184

26 апреля 2023 г., 17:59:39

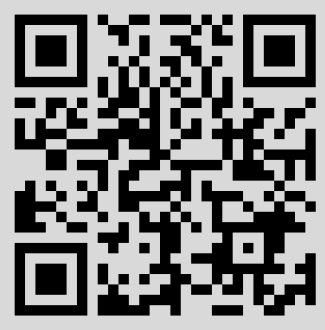


УДК 517.956.3

\title{
КОРРЕКТНОСТЬ ЛОКАЛЬНОЙ КРАЕВОЙ ЗАДАЧИ В ЦИЛИНДРИЧЕСКОЙ ОБЛАСТИ ДЛЯ МНОГОМЕРНОГО ВОЛНОВОГО УРАВНЕНИЯ
}

\author{
C. А. Алдашев \\ Актюбинский государственный университет им. К. Жубанова, \\ 030000, Казахстан, Актобе, ул. Бр. ЖКубановых, 263. \\ E-mail: aldash51@mail.ru
}

\begin{abstract}
Показана однозначная разрешимость локальной краевой задачи в иилиндрической области для многомерного волнового уравнения, которая является обобщением задач Дирихле и Пуанкаре. Получен критерий единственности регулярного решения.
\end{abstract}

Ключевые слова: многомерное волновое уравнение, иилиндрическая область, локальная краевая задача, разрешимость, единственность решения.

В работе показана однозначная разрешимость локальной краевой задачи в цилиндрической области для многомерного волнового уравнения, которая является обобщением задач Дирихле и Пуанкаре. Получен критерий единственности регулярного решения.

Для двумерного пространства в [1] было показано, что одна из фундаментальных задач математической физики - изучение поведения колебаний струны - некорректна, когда краевые условия заданы на всей границе области. Как замечено в [2,3], задача Дирихле некорректна не только для волнового уравнения, но и для общих гиперболических уравнений. В [4] показано, что решение задачи Дирихле существует в прямоугольных областях. В дальнейшем эта задача исследовалась методами функционального анализа [5], которые сложно применить в приложениях.

Для трёхмерного пространства получены теоремы единственности решения задачи Дирихле для строго гиперболических уравнений [6,7], а в [8,9] доказана корректность задач Дирихле и Пуанкаре для многомерного волнового уравнения.

Пусть $D_{\alpha}$ - цилиндрическая область евклидова пространства $E_{m+1}$ точек $\left(x_{1}, x_{2}, \ldots, x_{m}, t\right)$, ограниченная цилиндром $\Gamma=\{(x, t):|x|=1\}$, плоскостями $t=\alpha>0$ и $t=0$, где $|x|$ 一 длина вектора $x=\left(x_{1}, x_{2}, \ldots, x_{m}\right)$. Части этих поверхностей, образующих границу $\partial D_{\alpha}$ области $D_{\alpha}$, обозначим через $\Gamma_{\alpha}, S_{\alpha}$, $S_{0}$ соответственно.

В области $D_{\alpha}$ рассмотрим многомерное волновое уравнение

$$
\Delta_{x} u-u_{t t}=0
$$

где $\Delta_{x}$ - оператор Лапласа по переменным $x_{1}, \ldots, x_{m}, m \geqslant 2$.

В дальнейшем для удобства перейдём от декартовых координат $x_{1}, x_{2}, \ldots$, $x_{m}, t$ к сферическим $r, \theta_{1}, \theta_{2}, \ldots, \theta_{m-1}, t, r \geqslant 0,0 \leqslant \theta_{1}<2 \pi, 0 \leqslant \theta_{i} \leqslant \pi$, $i=2,3, \ldots, m-1$.

Серик Аймурзаевич Алдашев (д.ф.-м.н., проф.), директор, институт прикладной математики и информатики. 
Рассмотрим следующую локальную краевую задачу.

ЗАДача 1. Найти решение уравнения (1) в области $D_{\alpha}$ из класса $C\left(\bar{D}_{\alpha}\right) \cap$ $C^{1}\left(D_{\alpha} \cup S_{0}\right) \cap C^{2}\left(D_{\alpha}\right)$, удовлетворяющее краевым условиям

$$
\left.u\right|_{S_{\alpha}}=\varphi_{1}(r, \theta),\left.\quad u\right|_{\Gamma_{\alpha}}=\phi_{1}(t, \theta),\left.\quad\left(\beta u+\gamma u_{t}\right)\right|_{S_{0}}=\varphi_{2}(r, \theta),
$$

где $\beta, \gamma=\mathrm{const}, \beta^{2}+\gamma^{2} \neq 0$, которая является обобщением задач Дирихле $(\gamma=0)$ и Пуанкаре $(\beta=0)$.

Пусть $\left\{Y_{n, m}^{k}(\theta)\right\}$ - система линейно независимых сферических функций порядка $n, 1 \leqslant k \leqslant k_{n},(m-2) ! n ! k_{n}=(n+m-3) !(2 n+m-2), \theta=\left(\theta_{1}, \ldots, \theta_{m-1}\right)$, $W_{2}^{l}(l=0,1, \ldots)$ - пространства Соболева.

Имеют место следующие утверждения [10].

Лемма 1. Пусть $f(r, \theta) \in W_{2}^{l}(S)$. Если $l \geqslant m-1$, то ряд

$$
f(r, \theta)=\sum_{n=0}^{\infty} \sum_{k=1}^{k_{n}} f_{n}^{k}(r) Y_{n, m}^{k}(\theta)
$$

а также ряды, полученные из него дифберенцированием порядка $p \leqslant l-m+1$, сходятся абсолютно и равномерно.

Лемма 2. Для того чтоби $f(r, \theta) \in W_{2}^{l}(S)$, необходимо и достаточно, чтобы коэфбициенты ряда (2) удовлетворяли неравенствам

$$
\left|f_{0}^{1}(r)\right| \leqslant c_{1}, \quad \sum_{n=1}^{\infty} \sum_{k=1}^{k_{n}} n^{2 l}\left|f_{n}^{k}(r)\right|^{2} \leqslant c_{2}, \quad c_{1}, c_{2}=\text { const. }
$$

Через $\bar{\varphi}_{1 n}^{k}(r), \psi_{n}^{k}(t), \bar{\varphi}_{2 n}^{k}(r)$ обозначим коэффициенты разложения ряда $(2)$, соответственно функций $\varphi_{1}(r, \theta), \psi(t, \theta), \varphi_{2}(r, \theta)$.

Tеорема 1. Пусть $\varphi_{1}(r, \theta) \in W_{2}^{l}\left(S_{\alpha}\right), \psi(t, \theta) \in W_{2}^{l}\left(\Gamma_{\alpha}\right), \varphi_{2}(r, \theta) \in W_{2}^{l}\left(S_{0}\right)$, $l>3 m / 2 u$

$$
\beta \sin \mu_{s, n} \alpha \neq \gamma \cos \mu_{s, n} \alpha, \quad s=1,2, \ldots
$$

Тогда задача 1 однозначно разрешима, где $\mu_{s}$ - положительные нули функиий Бесселя первого рода $J_{n+(m-2) / 2}(z)$.

Доказательство. В сферических координатах уравнение (1) имеет вид

$$
\begin{gathered}
u_{r r}+\frac{m-1}{r} u_{r}-\frac{1}{r^{2}} \delta u-u_{t t}=0, \\
\delta \equiv-\sum_{j=1}^{m-1} \frac{1}{g_{j} \sin ^{m-j-1} \theta_{j}} \frac{\partial}{\partial \theta_{j}}\left(\sin ^{m-j-1} \theta_{j} \frac{\partial}{\partial \theta_{j}}\right), \\
g_{1}=1, \quad g_{j}=\left(\sin \theta_{1} \cdots \sin \theta_{j-1}\right)^{2}, \quad j>1 .
\end{gathered}
$$

Известно [10], что спектр оператора $\delta$ состоит из собственных чисел $\lambda_{n}=$ $=n(n+m-2), n=0,1, \ldots$, каждому из которых соответствует $k_{n}$ ортонормированных собственных функций $Y_{n, m}^{k}(\theta)$. 
Так как искомое решение задачи $D$ принадлежит классу $C\left(\bar{D}_{\alpha}\right) \cap C^{2}\left(D_{\alpha}\right)$, его можно искать в виде

$$
u(r, \theta, t)=\sum_{n=0}^{\infty} \sum_{k=1}^{k_{n}} \bar{u}_{n}^{k}(r, t) Y_{n, m}^{k}(\theta)
$$

где $\bar{u}_{n}^{k}(r, t)-$ функции, подлежащие определению.

Подставляя (5) в (4), используя ортогональность сферических функций $Y_{n, m}^{k}(\theta)($ см. [10]), будем иметь

$$
\bar{u}_{n r r}^{k}+\frac{m-1}{r} \bar{u}_{n r}^{k}-\bar{u}_{n t t}^{k}-\frac{\lambda_{n}}{r^{2}} \bar{u}_{n}^{k}=\mu \bar{u}_{n}^{k}, \quad k=1,2, \ldots, k_{n}, n=0,1, \ldots,
$$

при этом первое условие краевых условий (2) с учётом леммы 1 запишется в виде

$$
\begin{array}{rlrl}
\bar{u}_{n}^{k}(r, \alpha) & =\bar{\varphi}_{1 n}^{k}(r), & \bar{u}_{n}^{k}(1, t)=\psi_{n}^{k}(t), \\
\beta \bar{u}_{n}^{k}(r, 0)+\gamma \bar{u}_{n t}^{k}(r, 0) & =\bar{\varphi}_{2 n}^{k}(r), \quad k=1,2, \ldots k_{n}, n=0,1, \ldots
\end{array}
$$

Произведя замену $\bar{v}_{n}^{k}(r, t)=\bar{u}_{n}^{k}(r, t)-\psi_{n}^{k}(t)$ в соотношениях $(6),(7)$, получим

$$
\begin{gathered}
\bar{v}_{n r r}^{k}+\frac{m-1}{r} \bar{v}_{n r}^{k}-\frac{\bar{\lambda}_{n}}{r^{2}} \bar{v}_{n}^{k}-\bar{v}_{n t t}^{k}=\bar{f}_{n}^{k}(r, t), \\
\bar{v}_{n}^{k}(r, \alpha)=\varphi_{1 n}^{k}(r), \bar{v}_{n}^{k}(1, t)=0, \quad \beta \bar{v}_{n}^{k}(r, 0)+\gamma \bar{v}_{n t}^{k}(r, 0)=\varphi_{2 n}^{k}(r), \\
\bar{f}_{n}^{k}(r, t)=\psi_{n r r}^{k}+\frac{\lambda_{n}}{r^{2}} \psi_{n}^{k}, \quad \varphi_{1 n}^{k}(r)=\bar{\varphi}_{1 n}^{k}(r)-\psi_{n}^{k}(\alpha), \\
\varphi_{2 n}^{k}(r)=\bar{\varphi}_{2 n}^{k}(r)-\beta \psi_{n}^{k}(0)-\gamma \psi_{n t}^{k}(0), \quad k=1,2, \ldots, k_{n}, n=0,1, \ldots
\end{gathered}
$$

После замены $\bar{v}_{n}^{k}(r, t)=r^{(1-m) / 2} v_{n}^{k}(r, t)$ задача $(8),(9)$ приводится к следующему виду:

$$
\begin{gathered}
L v_{n}^{k} \equiv v_{n r r}^{k}-v_{n t t}^{k}+\frac{\bar{\lambda}_{n}}{r^{2}} v_{n}^{k}=f_{n}^{k}(r, t), \\
v_{n}^{k}(r, \alpha)=\tilde{\varphi}_{1 n}^{k}(r), \quad v_{n}^{k}(1, t)=0, \quad \beta v_{n}^{k}(r, 0)+\gamma v_{n t}^{k}(r, 0)=\tilde{\varphi}_{2 n}^{k}(r), \\
\bar{\lambda}_{n}=\left((m-1)(3-m)-4 \lambda_{n}\right) / 4, \quad f_{n}^{k}(r, t)=r^{(m-1) / 2} \bar{f}_{n}^{k}(r, t), \\
\tilde{\varphi}_{j n}^{k}(r)=r^{(m-1) / 2} \varphi_{j n}^{k}(r), \quad j=1,2 .
\end{gathered}
$$

Решение задачи (10), (11) представляется так:

$$
v_{n}^{k}(r, t)=v_{1 n}^{k}(r, t)+v_{2 n}^{k}(r, t),
$$

где $v_{1 n}^{k}(r, t)$ - решение задачи

$$
\begin{gathered}
L v_{1 n}^{k}=f_{n}^{k}(r, t), \\
v_{1 n}^{k}(r, \alpha)=0, \quad v_{1 n}^{k}(1, t)=0, \quad \beta v_{1 n}^{k}(r, 0)+\gamma v_{1 n t}^{k}(r, 0)=0,
\end{gathered}
$$


а $v_{2 n}^{k}(r, t)$ - решение задачи

$$
\begin{gathered}
L v_{2 n}^{k}=0, \\
v_{2 n}^{k}(r, \alpha)=\tilde{\varphi}_{1 n}^{k}(r), \quad v_{2 n}^{k}(1, t)=0, \quad \beta v_{1 n}^{k}(r, 0)+\gamma v_{2 n t}^{k}(r, 0)=\tilde{\varphi}_{2 n}^{k}(r) .
\end{gathered}
$$

Решение вышеуказанных задач будем искать в виде

$$
v_{n}^{k}(r, t)=\sum_{s=1}^{\infty} R_{s}(r) T_{s}(t),
$$

при этом

$$
\begin{gathered}
f_{n}^{k}(r, t)=\sum_{s=1}^{\infty} a_{s, n}(t) R_{s}(r), \quad \tilde{\varphi}_{1 n}^{k}(r)=\sum_{s=1}^{\infty} b_{s, n} R_{s}(r), \\
\tilde{\varphi}_{2 n}^{k}(r)=\sum_{s=1}^{\infty} e_{s, n} R_{s}(r) .
\end{gathered}
$$

Подставляя (17) в (13), (14), с учётом (18) получим

$$
\begin{gathered}
R_{s r r}+\frac{\bar{\lambda}_{n}}{r^{2}} R_{s}+\mu R_{s}=0, \quad 0<r<1, \\
R_{s}(1)=0, \quad\left|R_{s}(0)\right|<\infty \\
T_{s t t}+\mu T_{s}(t)=-a_{s, n}(t), \quad 0<t<\alpha, \\
T_{s}(\alpha)=0, \quad \beta T_{s}(0)+\gamma T_{s t}(0)=0 .
\end{gathered}
$$

Согласно [11], ограниченным решением задачи (19), (20) является

$$
R_{s}(r)=\sqrt{r} J_{\nu}\left(\mu_{s, n} r\right)
$$

где $\nu=(n+(m-2)) / 2, \mu=\mu_{s, n}^{2}$.

Общее решение уравнения (21) представимо в виде

$$
\begin{aligned}
T_{s, n}(t)=c_{1 s} \cos \mu_{s, n} t+c_{2 s} \sin \mu_{s, n} t+ & \frac{\cos \mu_{s, n} t}{\mu_{s, n}} \int_{0}^{t} a_{s, n}(\xi) \sin \mu_{s, n} \xi d \xi- \\
& -\frac{\sin \mu_{s, n} t}{\mu_{s, n}} \int_{0}^{t} a_{s, n}(\xi) \cos \mu_{s, n} \xi d \xi
\end{aligned}
$$

где $c_{1 s}, c_{2 s}$ - произвольные постоянные [10].

Удовлетворив условиям (22), получим систему алгебраических уравнений

$$
\left\{\begin{aligned}
c_{1 s} \cos \mu_{s, n} \alpha+c_{2 s} \sin \mu_{s, n} \alpha= & -\frac{\cos \mu_{s, n} \alpha}{\mu_{s, n}} \int_{0}^{\alpha} a_{s, n}(\xi) \sin \mu_{s, n} \xi d \xi+ \\
& +\frac{\sin \mu_{s, n} \alpha}{\mu_{s, n}} \int_{0}^{\alpha} a_{s, n}(\xi) \cos \mu_{s, n} \xi d \xi \\
\beta c_{1 s}+\gamma \mu_{s} c_{2 s}=0, &
\end{aligned}\right.
$$

которое имеет единственное решение, если выполняется условие (3). 
Подставляя (23) в (18), определяем

$$
\begin{gathered}
r^{-1 / 2} f_{n}^{k}(r, t)=\sum_{s=1}^{\infty} a_{s, n}(t) J_{\nu}\left(\mu_{s, n} r\right), \quad r^{-1 / 2} \tilde{\varphi}_{1 n}^{k}(r)=\sum_{s=1}^{\infty} b_{s, n} J_{\nu}\left(\mu_{s, n} r\right), \\
r^{-1 / 2} \tilde{\varphi}_{2 n}^{k}(r)=\sum_{s=1}^{\infty} e_{s, n} J_{\nu}\left(\mu_{s, n} r\right), \quad 0<r<1 .
\end{gathered}
$$

Ряды (26) - разложения в ряды Фурье-Бесселя [12], если

$$
\begin{gathered}
a_{s, n}(t)=2\left[J_{\nu+1}\left(\mu_{s, n}\right)\right]^{-2} \int_{0}^{1} \sqrt{\xi} f_{n}^{k}(\xi, t) J_{\nu}\left(\mu_{s, n} \xi\right) d \xi \\
b_{s, n}=2\left[J_{\nu+1}\left(\mu_{s, n}\right)\right]^{-2} \int_{0}^{1} \sqrt{\xi} \tilde{\varphi}_{1 n}^{k}(\xi) J_{\nu}\left(\mu_{s, n} \xi\right) d \xi \\
e_{s, n}=2\left[J_{\nu+1}\left(\mu_{s, n}\right)\right]^{-2} \int_{0}^{1} \sqrt{\xi} \tilde{\varphi}_{2 n}^{k}(\xi) J_{\nu}\left(\mu_{s, n} \xi\right) d \xi
\end{gathered}
$$

$\mu_{s}, s=1,2, \ldots$ - положительные нули функций Бесселя $J_{\nu}(z)$, расположенные в порядке возрастания их величины.

Из (23), (24) получим решение задачи (13), (14) в виде

$$
v_{1 n}^{k}(r, t)=\sum_{s=1}^{\infty} \sqrt{r} T_{s, n}(t) J_{\nu}\left(\mu_{s, n} r\right)
$$

где $a_{s, n}(t), c_{1 s}, c_{2 s}$ определяются из $(27),(25)$.

Далее, подставляя (23) в (15) и (16), с учётом (18) будем иметь задачу

$$
\begin{gathered}
V_{s t t}+\mu_{s}^{2} V_{s}=0, \\
V_{s}(\alpha)=b_{s, n}, \beta V_{s}(0)+\gamma V_{s t}(0)=e_{s} .
\end{gathered}
$$

Общее решение уравнения (30) имеет вид

$$
V_{s, n}(t)=c_{1 s}^{\prime} \cos \mu_{s, n} t+c_{2 s}^{\prime} \sin \mu_{s, n} t
$$

где $c_{1 s}^{\prime}, c_{2 s}^{\prime}$ - произвольные постоянные. Удовлетворив условиям (31), получим

$$
\left\{\begin{array}{l}
c_{1 s}^{\prime} \cos \mu_{s, n} \alpha+c_{2 s}^{\prime} \sin \mu_{s, n} \alpha=b_{s, n} \\
\beta c_{1 s}^{\prime}+\gamma \mu_{s} c_{2 s}^{\prime}=e_{s, n}
\end{array}\right.
$$

Из (23), (32) имеем

$$
v_{2 n}^{k}(r, t)=\sum_{s=1}^{\infty} \sqrt{r} V_{s, n}(t) J_{\nu}\left(\mu_{s, n} r\right)
$$

где $b_{s}, e_{s}, c_{1 s}^{\prime}, c_{2 s}^{\prime}$ находятся из (28), (33).

Таким образом, из (5), (12) следует, что решением задачи 1 является ряд

$$
u(r, \theta, t)=\sum_{n=0}^{\infty} \sum_{k=1}^{k_{n}}\left\{\psi_{n}^{k}(t)+r^{(1-m) / 2}\left[v_{1 n}^{k}(r, t)+v_{2 n}^{k}(r, t)\right]\right\} Y_{n, m}^{k}(\theta)
$$

где $v_{1 n}^{k}(r, t), v_{2 n}^{k}(r, t)$ определяются из $(29),(34)$.

Отметим следующие свойства нулей функций Бесселя (см. [12, 13]): 
$\left.1^{\circ}\right)$ если $\mu_{\nu, 1}, \mu_{\nu, 2}, \ldots$ - положительные нули функций $J_{\nu}(z)$, упорядоченные по возрастанию значений, то

$$
0<\mu_{\nu, 1}<\mu_{\nu+1,1}<\mu_{\nu, 2}<\mu_{\nu+1,2}<\mu_{\nu, 3}<\ldots, \quad \nu>-1
$$

$2^{\circ}$ ) если $\mu_{\nu}, \mu_{\nu}^{\prime}, \mu_{\nu}^{\prime \prime}$ являются наименьшими положительными нулями функций $J_{\nu}(z), J_{\nu}^{\prime}(z), J_{\nu}^{\prime \prime}(z)$ соответственно, то справедливы неравенства

$$
\begin{gathered}
\sqrt{\nu(\nu+2)}<\mu_{\nu}<\sqrt{2(\nu+1)(\nu+3)}, \quad \nu>0, \\
\sqrt{\nu(\nu+2)}<\mu_{\nu}^{\prime}<\sqrt{2 \nu(\nu+1)}, \quad \nu>0 \\
\sqrt{\nu(\nu-1)}<\mu_{\nu}^{\prime \prime}<\sqrt{\left(\nu^{2}-1\right)}, \quad \nu>1
\end{gathered}
$$

и формулы

$$
\begin{gathered}
\sin z=z\left(1-z \sum_{n=1}^{\infty}\left(4 n^{2}-1\right)^{-1}\left[J_{n}(n z)\right]^{2}\right) \\
J_{\nu}(z)=\sqrt{\frac{2}{\pi z}} \cos \left(z-\frac{\pi}{2} \nu-\frac{\pi}{4}\right)+\mathrm{O}\left(\frac{1}{z^{3 / 2}}\right), \quad \nu \geqslant 0, \\
2 J_{\nu}^{\prime}(z)=J_{\nu-1}(z)-J_{\nu+1}(z) .
\end{gathered}
$$

Учитывая (36)-(38) и применяя признак Даламбера, можно показать, что ряды (29), (34) и продифференцированные ряды сходятся абсолютно и равномерно.

Далее, используя формулы (38) и оценки [12]

$$
\left|k_{n}\right| \leqslant c_{1} n^{m-2}, \quad\left|\frac{\partial^{q}}{\partial \theta_{j}^{q}} Y_{n, m}^{k}(\theta)\right| \leqslant c_{2} n^{m / 2-1+q},
$$

где $j=1,2, \ldots, m-1, q=0,1, \ldots$, a также леммы, ограничения на заданные функции $\varphi_{1}(r, \theta), \varphi_{2}(r, \theta), \psi(t, \theta)$, аналогично $[8,9]$ показывается, что полученное решение в виде ряда (35) и дважды продифференцированные ряды сходятся абсолютно и равномерно. Это означает, что решение (35) принадлежит классу $C\left(\bar{D}_{\alpha}\right) \cap C^{1}\left(D_{\alpha} \cup S_{0}\right) \cap C^{2}\left(D_{\alpha}\right)$.

Теорема 2. Решение задачи 1 единственно тогда и толъко тогда, когда выполняется условие (3).

Доказательств о. Если выполняется условие (3), то из теоремы 1 вытекает единственность решения задачи 1. Пусть теперь условие (3) нарушено хотя бы для одного $s=p$. Тогда нетривальным решением однородной задачи, соответствующей задаче 1 , является функция

$$
u(r, \theta, t)=\sum_{n=0}^{\infty} \sum_{k=1}^{k_{n}} n^{-l} r^{(2-m) / 2}\left[\beta \sin \mu_{p} t-\gamma \mu_{p} \cos \mu_{p} t\right] J_{n+(m-2) / 2}\left(\mu_{p} r\right) Y_{n, m}^{k}(\theta)
$$

при этом из (38), (39) следует, что она принадлежит искомому классу, если $l>3 m / 2$. 
В заключение отметим, что в [14] для уравнения (1) внутри характеристической области приведены корректные постановки задач Дирихле и Пуанкаре.

\section{БИБЛИОГРАФИЧЕСКИЙ СПИСОК}

1. Hadamard J. Sur les problèmes aux dérivés partielles et leur signification physique// Princeton University Bulletin, 1902. Vol. 13. Pp. 49-52.

2. Бицадзе A. В. Уравнения смешанного типа. М.: АН CCCP, 1959. 164 с. [Bitsadze A. V. Mixed-Type Equations. Moscow: Akad. Nauk USSR, 1959. 164 pp.]

3. Нахушев А. М. Задачи со смещением для уравнений в частных производных. М.: Наука, 2006. 287 с. [Nakhushev A. M. Problems with Displacement for a Partial Differential Equation. Moscow: Nauka, 2006. 287 pp.]

4. Bourgin D. G., Duffin R. The Dirichlet problem for the virbrating string equation // Bull. Amer. Math. Soc., 1939. Vol. 45. Pp. 851-858.

5. Fox D. W., Pucci C. The Dirichlet problem for the wave equation // Ann. Mat. Pura Appl. (4), 1958. Vol. 46. Pp. 155-182.

6. Нахушев А. М. Критерий единственности задачи Дирихле для уравнения смешанного типа в цилиндрической области // Диффер. уравн., 1970. Т. 6, №1. С. 190-191. [Nakhushev A. M. Uniqueness Criterion for the Dirichlet Problem for an Equation of Mixed Type in a Cylindrical Domain // Differ. Uravn., 1970. Vol. 6, no. 1. Pp. 190-191].

7. Dunninger D. R.; Zachmanoglou E. C. The condition for uniqueness of the Dirichlet problem for hyperbolic equations in cylindrical domains // J. Math. Mech., 1969. Vol. 18. Pp. 763-766.

8. Aldashev S. A. The well-posedness of the Dirichlet problem in the cylindric domain for the multidimensional wave equation // Math. Probl. Eng, 2010. Vol. 2010, 653215. 7 pp.

9. Алдашев C. А. Корректность задачи Пуанкаре в цилиндрической области для многомерного волнового уравнения / Современная математика и ее приложения, Т. 67, Уравнения с частными производными, 2010. С. 28-32; Aldashev S. A. The well-posedness of the Poincaré problem in the cylindric domain for the higher-dimensional wave equation // J. Math. Sci. (N. Y.), 2011. Vol.173, no. 2. Pp. 150-154.

10. Михлин С. Г. Многомерные сингулярные интегралы и интегральные уравнения. М.: Физматлит, 1962. 254 с. [Mihlin S. G. Higher-dimensional singular integrals and integral equations. Moscow: Fizmatlit, 1962. 254 pp.]

11. Камке Э. Справочник по обыкновенным дифференциальным уравнениям. М.: Наука, 1965. 703 c. [Kamke É. Manual of ordinary differential equations. Moscow: Nauka, 1965. 703 pp.]

12. Erdélyi A., Magnus W., Oberhettinger F., Tricomi F. G. Higher transcendental functions. Vol. II / ed. H. Bateman. New York - Toronto - London: McGraw-Hill Book Co, Inc., 1953. 396 pp.; русск. пер.: Бейтмен Г., Эрдейи А. Высшие трансцендентные функции. В 3-х т. Т. 2: Функции Бесселя, функции параболического цилиндра, ортогональные многочлены. М.: Наука, 1974. 295 с.

13. Тихонов A.Н., Самарский A. А. Уравнения математической физики. М.: Наука, 1966. 724 c. [Tihonov A. N., Samarskiy A. A. Equations of mathematical physics. Moscow: Nauka, 1966. 724 pp.]

14. Алдашев C. А. Задачи Дирихле и Пуанкаре для многомерного волнового уравнения // Изв. НАН РК, Сер. физ.-мат., 2010. №1. С. 3-6. [Aldashev S. A. Dirichlet and Poincaré problems for higher-dimensional wave equation // Izv. NAN RK, Ser. Fiz.-Mat., 2010. no. 1. Pp. 3-6]. 
MSC: 35L05; 35R25

THE WELL-POSEDNESS OF THE LOCAL BOUNDARY VALUE

PROBLEM IN A CYLINDRIC DOMAIN FOR THE MULTI-DIMENSIONAL WAVE EQUATION

\section{S. A. Aldashev}

Aktobe State University after K. Zhubanov,

263, Zhubanov Bruthers st., Aktobe, Kazakhstan, 030000.

E-mail: aldash51@mail.ru

This paper proves the unique solvability of the local boundary value problem in a cylindric domain for the multi-dimensional wave equation, which is the generalization of the Dirichlet and Poincare problems. We also obtain the criterion for the uniqueness of the regular solution.

Key words: multi-dimensional wave equation, cylindrical domain, local boundary value problem, solvability, uniqueness of solutions.

Original article submitted 10/V/2012; revision submitted 12/VIII/2012.

Serik A. Aldashev (Dr. Sci. (Phys. \& Math.)), Director, Institute of Applied Mathematics. 